\title{
Automated segmentation of wood fibres in micro CT images of paper
}

\author{
Y. Sharma ${ }^{1,2}$, A.B. Phillion ${ }^{2 *}$ D. M. Martinez ${ }^{1,3}$ \\ ${ }^{1}$ Department of Mechanical Engineering, The University of British Columbia, Vancouver, \\ Canada. \\ ${ }^{2}$ School of Engineering, Okanagan campus, The University of British Columbia, Kelowna, \\ Canada. \\ ${ }^{3}$ Department of Chemical and Biological Engineering, The University of British Columbia, \\ Vancouver, Canada \\ *Corresponding Author- Dr. Andre Phillion \\ Assistant Professor, School of Engineering, UBC Okanagan \\ T: +1 (250) 807-9403, E: andre.phillion@ubc.ca
}

Keywords- Fibre segmentation, X-ray tomographic microscopy, micro CT, Paper, Fibre properties

\begin{abstract}
A novel algorithm has been developed and validated to isolate individual papermaking fibres in $\mu \mathrm{CT}$ images of paper handsheets as a first step to characterize the structure of paper. The three step fibre segmentation algorithm segments the papermaking fibres by (i) tracking the hollow inside the fibres via a modified connected component methodology, (ii) extracting the fibre walls using a distance transform and (iii) labeling the fibres through collapsed sections by a final refinement step. Further, post processing algorithms have been developed to calculate the length and coarseness of the segmented fibres. The Fibre Segmentation algorithm is the first ever reported method for the automated and robust segmentation of the tortuous 3D morphology of papermaking fibres within microstructural images of paper handsheets. The method is not limited to papermaking fibres, but can be applied to any material consisting of tortuous and hollow fibres.
\end{abstract}




\section{Introduction}

Several classes of materials, such as ceramics, composites, textiles, paper, and foams consist of a dense network arrangement of tubular fibres. Industrially, there is a widespread interest to precisely characterize the fibres at the micron scale in order to link the macro-scale properties such as strength, stiffness, and permeability with the structure of the fibrous network. While $\mathrm{X}$-ray micro computed tomography $(\mu \mathrm{CT}), 3 \mathrm{D}$ non-destructive imaging technique, has the potential to reveal the 3D network microstructure; advanced image analysis techniques are required in order to gain meaningful insight into the properties of the material. The first and often most challenging task in the analysis of fibres is the isolation of each individual fibre in the 3D network, also known as fibre segmentation. Fibre segmentation gives access to the properties at the fibre level such as the length, cross-section area, and orientation, as well as the properties at the network level including the coordination number of the fibre-fibre bonds. Although segmentation is a widely studied topic, most work-to-date has focused on the application of semiautomatic techniques to separate only a few phases in the $3 \mathrm{D}$ images. The characterization of a fibrous network requires automated and robust segmentation of a large number of tortuous 3D fibres in large datasets.

The material studied in this work is paper, a layered network of papermaking fibres, fibre fragments, and possibly fillers. Figure 1 shows a 3D image of a paper handsheet obtained using $\mu \mathrm{CT}$ and a corresponding $2 \mathrm{D}$ section. The white regions in the $2 \mathrm{D}$ slice represent the fibre walls, the hollows inside them are called lumen, and the remaining empty space is air. The fibres have a tortuous morphology, and may be kinked, twisted, broken or collapsed. An example of a pore is given in Figure 1(B). The fibres also form a dense network that is packed together and is continuous as shown in Figure 1(B). Owing to these reasons, conventional segmentation techniques are inadequate for fibre segmentation. 
An alternative approach for segmenting papermaking fibres and other complex tubular structures is based on using the lumen as markers for fibre segmentation since they are separated from the background by means of thick fibre walls. Aronsson (2002) applied an ordered Region Growing (Adams \& Bischof, 1994) algorithm to segment papermaking fibres from a 3D dataset obtained by SEM and microtomy, in which the lumens were grown from user-selected seed points. Axelsson (2007) presented a similar method for tracking of fibres in their direction of local orientation. Sevensson and Aronsson (2003) later extended their previous work to include the cracks in the fibre walls based on fuzzy connectedness (Udupa \& Samarasekera, 1996). While these methods have proven useful in segmenting hollow fibres, they require the user to select a number of seed points for each fibre in order to initiate the segmentation. Thus the number of fibres that can be segmented is limited.

Graph-based methods have also been used to segment papermaking fibres. Wernersson, Brun \& Hendriks (2009) showed that a fibrous network structure could be converted to a mathematical graph where each edge represents a free fibre segment, and clusters represent fibre-fibre bonds. Individual fibres were then segmented based on the graph representation. Yang \& Lindquist (2000) connected fibre fragments across clusters utilizing a graph and then traced fibres in 3D data of a synthetic fibre mat. Lux, Delisee \& Thibault (2006) obtained curve skeletons of fibres, and then segmented synchrotron tomography images of wood-based fibrous materials. Sandau \& Ohser (2007) presented advanced morphological transforms to trace the fibres through intersections in order to identify crossing points. The graph-based methods increase the degree of fibre segmentation within a 3D $\mu \mathrm{CT}$ image as compared to the regiongrowing methods, but do not preserve the topology of the fibres since they are based on identifying the medial axes of the fibres. Knowledge of topology is important when examining the mechanical behaviour of the fibrous network. 
Other researchers have attempted to automate the segmentation by automatically identifying lumen cross-sections (Aronsson, 2002; Kontschieder et al. 2010; Bache-Wiig \& Henden, 2005). Donoser \& Bischof (2006) developed an efficient algorithm to track lumen in 3D by simultaneously detecting and tracking the lumen cross-sections as Maximally Stable Extremal Regions (Matas et al., 2004). Kontschieder et. al. (2010) demonstrated the successful identification of more than $80 \%$ of fibre cross sections in 2D microtomy images through the use of a novel shape descriptor (Donoser et al., 2009; Lu et al., 2009). Aronsson (2002) automated the process by first detecting the lumen cross sections as circular regions, and then applying further constraints on area and curvature to enable segmentation. Bache-Wiig \& Henden (2005) extended this idea by applying additional constraints related to bending energy, eccentricity, and convexity to reduce the occurrence of false positives.

As can be seen, a number of different methods have been proposed to segment papermaking fibres using their lumen as the starting point. However, the methods proposed thus far require either significant user input, or are too computationally complex for large-scale fibre segmentation in 3D. In this work, a new method is presented that will allow for robust and fully automated segmentation of hollow tubular fibres present in large 3D datasets. First, the method itself is presented. Then, the algorithm is benchmarked against a series of specimens with known geometry. Finally, the capabilities and limitations of the new method are discussed by examining the results from a sample paper handsheet.

\section{Methods}

\subsection{Materials}

The paper sample used to analyze the capabilities of the new fibre segmentation algorithm, with a basis weight of $59 \mathrm{~g} / \mathrm{m}^{2}$, was produced using a British handsheet maker at UBC's Pulp and Paper Centre from an NBSK pulp obtained from Canfor Pulp (Prince George, BC, 
Canada). The paper samples were freeze-dried to increase the percentage of fibres with uncollapsed lumens within the paper handsheet.

\subsection{Experimental Procedure}

X-ray tomographic microscopy was performed on a paper handsheet in order to acquire the 3D datasets that require fibre segmentation. The tomographic imaging was performed using a Zeiss MicroXCT-400 X-ray instrument located at The University of British Columbia's Okanagan campus. For imaging purposes, the handsheet was first cut to a size of $1.0 \mathrm{~mm} \times 1.0 \mathrm{~mm}$ in cross-section and 250 microns thick, and then firmly secured to a sample holder. The X-ray radiographs were then acquired using a $40 \mathrm{kV}$ X-ray source, a $30 \mathrm{~s}$ exposure time, and 2500 radiographs spanning $360^{\circ}$. A long exposure time was required to offset the poor contrast at the fibre-air interfaces. From these radiographs, a 3D volume consisting of $2000 \times 2000 \times 300$ voxels was reconstructed with a resolution of $0.58 \mu \mathrm{m}$ per voxel side. Finally, a simple nonlinear diffusion filter (Wieckert et al., 1998) was applied for noise reduction and edge enhancement. An example is given in Figure 1 along with the $x, y, z$ coordinate system. Note that the same coordinate system will be used for all future reference in this work: the $x-y$ plane denotes a plane cut perpendicular to the papermaking "machine direction" and the $z$ direction is in the plane of the sheet.

\subsection{Image Analysis}

\subsubsection{Initial Segmentation}

Prior to segmenting individual fibres, the 3D image must first be segmented into two phases, a process known as binarization. For papermaking fibres, selection of the threshold for binarization is challenging due to the poor image contrast and streaking artefacts. Furthermore, statistical thresholding techniques (Sezgin \& Sankur, 2004) cannot be relied upon for threshold 
selection since it does not provide an accurate estimate of the fibre wall thickness of individual fibres.

A known quantity of the papermaking fibres is the basis weight, $w$, which is defined as the weight of fibres per unit area. The basis weight of a handsheet is given by

$$
w=\frac{m}{A}
$$

where $m$ is the mass of fibres in the sheet, and $A$ is the total surface area of the sheet. From the 3D image, the total mass of fibres present, $m$, is given by

$$
m=N(T) *(r)^{3} * \rho
$$

where $N(T)$ is the total number of voxels with grayscale value greater than $T, r$ is the voxel size of the acquired image and $\rho$ is the density of papermaking fibres. By combining equations (1) and (2), the basis weight can be estimated from the 3D image as

$$
w=\frac{N(T) *(r)^{3} * \rho}{A}
$$

The only free parameter in equation (3) is the threshold value, $T$, and thus an appropriate threshold value can be selected by comparing the calculated basis weight for a given value of $T$ against the measured experimental value.

\subsubsection{Fibre Segmentation}

The new algorithm for segmenting papermaking fibres and other tubular fibrous materials consists of three stages:

(i) Lumen Tracking - In this stage, the lumen are tracked throughout the 3D dataset using a modified connected components labeling algorithm.

(ii) Fibre walls Extraction - The fibre walls surrounding each tracked lumen are then labeled via a distance transform, individually segmenting each fibre. 
(iii) Second-level Segmentation - In this stage, regions with collapsed or crushed lumen are labelled and fibre fragments from Step 1 and Step 2 are merged.

\section{Lumen Tracking}

The purpose of the lumen tracking stage is to individually identify, in $3 \mathrm{D}$, the hollow at the centre of the papermaking fibres. The method takes advantage of the fact that although the lumen geometry is not closed in $3 \mathrm{D}$, owing to the presence of pores and end-points, the lumen cross-sections are closed 2D shapes in most of the cross-section slices of the dataset. A sequential algorithm has been written to track the lumen in 3D by connecting multiple lumen cross-sections segmented in successive 2D slices ( $x-y$ planes) based on their connectivity in the $\mathrm{z}$ direction while imposing additional constraints to prevent leakage of the contour through the cracks and pores in the fibre walls. First, 2D connected component labeling is applied to each cross-section slice in the binarized volume to segment lumen cross-sections. This will separate and identify most of the lumen cross-sections from the background. The exceptions are the lumens that are cracked, as they will be merged with background. Second, the lumen crosssections in successive slices are merged to create a 3D shape. Tracking of lumen is based on the assumption that the cross-sections in adjacent slices will have some overlapping points. This is true only for adjacent slices (or a few slices apart) as the fibres twist and turn at sharp angles. Based on the connectivity of points in adjacent slices, a lumen is built throughout its length by connecting 2D cross sections in the longitudinal direction. Figure 2 shows the merging of lumen cross-sections in three adjacent slices. The advantage of this method is that the topology of a lumen is preserved in its entirety. The major challenge in lumen tracking is in developing a mechanism to handle pores and fibre end-points that might appear in the 3D volume. Otherwise, a simple 3D connected component labeling could be used to segment the lumen. Figure 3(A) shows a typical result obtained after application of a 2D connected component labeling method to a $2 \mathrm{D}$ cross-section slice. In this figure, the blue region is the 
background, the black represents the fibre walls, and the coloured blobs represent the crosssections of lumen. As can be seen, lumens that are not perfectly isolated by fibre, such as the one in the upper left-hand corner of Figure 3(A), become merged with the background. The developed algorithm will identify pores and end-points by comparing the change in lumen cross-section area between subsequent slices. If a lumen, upon appearing very large in slice $k$, returns to its original size after $n$ slices then the defect is classified as a pore and the intersection of the lumen in slice $k+n$ with the background in the slices $k$ to $k+n-1$ is assigned as the lumen cross-section in those $n$ slices. The lumen then continues to grow normally. However, if the lumen continues to remain large beyond $n$ slices, the fibre is deemed to have terminated in slice $k-1$. The effectiveness of this method is demonstrated in Figure 3(B), where the lumen that was merged with the background by the 2D connected component labeling method in Figure 3(A) has been successively isolated. This method of tracking the lumen while taking care of pores enables for robust and completely autonomous lumen segmentation.

\section{Fibre Walls Extraction}

The lumen-tracking algorithm outlined in the previous section results in a complete threedimensional segmentation of the lumens in the volume. These segmented lumens are then used as markers to extract the fibre walls surrounding each lumen. The general rule applied for assigning fibre walls to lumens is that each voxel in the fibre phase assumes the label of the closest lumen. In order to preserve the topology and thickness of the papermaking fibre walls as well as to create the boundary at the touching points of the fibres, a method based on a Distance Transform has been developed. Specifically, a Distance Transform is sequentially performed on each 2D slice from the segmented lumen dataset obtained in the previous section. The newly created slices are then combined through a series of arithmetic operations with slices from the binarized image from Section 2.3.1 to extract the fibre wall cross-sections. The stepwise process is explained below. 


\section{Input: 2D Slices from Datasets $A$ (Segmented Lumen) and $B$ (Binarized Fibre Walls)}

Step \#1: Distance Transform Operation on $A$ : By applying a distance transform to $A$ giving $C$, all pixels belonging to the segmented lumens are given a value of zero while the remaining pixels are given a value corresponding to the distance from the closest lumen. In a second array, $I$, the value of each pixel in $A$ is replaced with a value that identifies its nearest lumen.

Step \#2: $\boldsymbol{B}$ X $C$ : Multiplying $B$ with $C$ giving $D$ eliminates the regions that do not correspond to fibre walls. All pixels belonging to lumen and the background are labeled as zero; while all pixels belonging to fibre walls are have a value representing the distance to the closest lumen.

Step \#3: Threshold Operation on $D$ : The thresholding of $D$ giving $E$ eliminates all fibre walls and fragments that do not surround a segmented lumen.

Step \#4: $\boldsymbol{E} \mathbf{X} I$ : Multiplying $E$ with $I$ giving $F$ assigns to each non-zero pixel in $E$ the identifier of its closest lumen.

\section{Output: 2D Slice $F$}

This process is repeated for every slice in datasets $A$ and $B$, and the corresponding output images are then stacked to create fibre walls in 3D that are individually segmented.

\section{Second Level Segmentation}

At this stage, every voxel in the dataset has been assigned a label that corresponds to one of: lumen, fibre wall or background. However, there is need to further refine the segmentation achieved thus far to account for the collapsed regions of papermaking fibres. These regions form during processing due to internal tension within the fibre, and cannot be segmented using the lumen tracking method. Instead, the segmentation of individual fibres terminates at the onset of collapse and a fibre with multiple crushed sections will appear as several fibres in the segmented dataset. An example is shown in Figure 4(A) and (B) In the second-level segmentation, the collapsed sections are segmented on the basis on connectivity in a manner similar to the Lumen 
Tracking algorithm. The difficulty is that the newly segmented fibres must grow in two directions in order to join pre-existing fibres, and may overlap with other segments. If this occurs, the fibres are merged as shown in Figure 4(C). By use of this methodology, the collapsed sections of fibres that were not labeled by the lumen tracking and fibre wall extraction algorithms are segmented and merged, resulting in an improved algorithm for automated fibre segmentation.

\subsubsection{Measurement of Fibre Properties}

The utility of a segmented 3D dataset is to quantify the fibrous structure. While many characteristic properties can be identified (Svensson \& Aronsson, 2003), two are examined in the present work in order to characterize fibres within a papermaking handsheet: Fibre Length and Fibre Coarseness.

\section{Fibre Length}

The length of a segmented fibre is determined by discretizing the 3D fibre into a sequence of chords connecting the centres of the fibre cross-sections in adjacent slices. The fibre crosssection centre in each slice is calculated individually using the k-means clustering method (Seber, 1984). The Euclidean length of the discrete chords is then summed to calculate the total length of a fibre.

\section{Fibre Coarseness}

Fibre coarseness is defined as the mass per unit length of the fibre.

$$
\mathrm{C}=\frac{\mathrm{m}}{\mathrm{L}}
$$

where $\mathrm{C}$ is the coarseness, $\mathrm{m}$ is the mass and $\mathrm{L}$ is the length of the fibre. Since the mass of a fibre is given by

$$
\mathrm{m}=\mathrm{V} * \rho
$$


where $\mathrm{V}$ is the volume of the fibre wall and $\rho$ is the density of the fibre, and the volume of a fibre is given by

$$
\mathrm{V}=\mathrm{N} * \mathrm{r}^{3}
$$

where $\mathrm{N}$ is the number of voxels labeled as the fibre wall and $\mathrm{r}$ is the resolution of the data, the coarseness of a segmented fibre can be determined as

$$
\mathrm{C}=\frac{\mathrm{N} * \mathrm{r}^{3} * \rho}{\mathrm{L}}
$$

Since, $\mathrm{r}$ and $\rho$ are known and $\mathrm{L}$ is the fibre length calculated above, measuring $\mathrm{C}$ only requires counting the voxels labeled as fibre walls.

\section{Algorithm Validation}

In order to validate the new fibre segmentation algorithm, a series of known test specimens were produced from thin-walled aluminum tubes to approximate the structure of paper handsheets. The aluminum tubes were given holes to represent pores, bent to simulate the tortuous and discontinuous morphology of papermaking fibres, and then embedded within epoxy resin as shown in Figure 5(A). Three test geometries of increasing complexity were created: (A) 50 straight hollow tubes $(\phi=1.57 \mathrm{~mm}, l=20 \mathrm{~mm}$ ); (B) 40 straight hollow tubes ( $\phi=3.24 \mathrm{~mm}, l=20 \mathrm{~mm}$ ) with either one or two holes in the walls of each cylinder; (C) 25 hollow tubes $(\phi=1.57 \mathrm{~mm}, l=20 \mathrm{~mm})$, bent in multiple locations and 25 hollow tubes $(\phi=3.24 \mathrm{~mm}$, $l=20 \mathrm{~mm}$ ), bent in multiple locations and containing multiple holes in the cylinder walls. Examples of the four types of tubes used in each test specimen are given in Figure 5(B). Each test specimen was first imaged using $\mu \mathrm{CT}$ and then analyzed using the fibre segmentation algorithm. A 2D image from test geometry $C$ is shown in Figure 5(C). As can be seen, the tubes have thick walls, representative of papermaking fibres, hollow inside representing lumens, and are touching each other thus forming a continuous network structure similar to that of paper. The final segmented dataset for test geometry $C$ is shown in Figure 5(D). 
The fibre segmentation algorithm has been validated by comparing the computed lengths and coarseness of the segmented tubes from the $\mu \mathrm{CT}$ datasets against the physical measurements. The results are given in Table 1 (length validation) and Table 2 (coarseness validation ${ }^{1}$ ). As can be seen, the length and coarseness of the tubes calculated using image analysis strongly correlate within $\sim 5 \%$ with the corresponding known values. Thus, the fibre segmentation algorithm is able to individually segment $3 \mathrm{D}$ tubular structures with tortuous and discontinuous morphologies similar to that of papermaking fibres within a network structure.

Referring to Table 1, it can also be seen that the difference between the calculated average tube length and the physically known value increases with increasing structural complexity, as does the standard deviation. This deviation in the length calculation is attributed to the alignment of the tubes within the test specimen. Specifically, if the tube does not lie perfectly perpendicular to the sectioning plane, the hollow of the tube near its end appears as an open section and the fibre segmentation algorithm interprets this as being the tube end. Examples of these open sections can be seen in Figure 5(C). This effect is more pronounced when the tubes are bent because the odds of having an out of plane tube section increases. It is expected that this type of error would be limited in paper handsheets since the fibres are primarily aligned in-plane due to the fabrication process. The deviations presented in Table 1 could also be due to the physical measurements since the measurements were made prior to bending the tubes to create the third and fourth tube types.

Referring to Table 2, it can be seen that the calculated coarseness of the tubes with a diameter of $1.57 \mathrm{~mm}$ (Test Geometries A and C) was reproducible with a small standard deviation while the calculated coarseness of the tubes with a diameter of $3.14 \mathrm{~mm}$ (Test Geometries B

\footnotetext{
${ }^{1}$ The physical coarseness of the aluminum tubes was calculated as $C=\pi\left(r_{2}^{2}-r_{1}^{2}\right) * \rho$, where $C$ is the coarseness, $r_{2}$ and $r_{1}$ are the outer and inner radii respectively, and $\rho$ is the density of aluminum.
} 
and C) was highly variable. This can be attributed to the fact that the holes within the tubes were created using a sharp object resulting in non-uniform removal of the material.

\section{Application of New Methodology to Paper Handsheets}

In order to demonstrate the capabilities of the new fibre segmentation tool for analysing tortuous fibres, the algorithm has been applied to a paper handsheet manufactured from NBSK pulp. Then, the fibre properties were measured and reported.

\subsection{Critical Analysis of Fibre Segmentation Methodology}

Below, each stage of the segmentation algorithm is critically analyzed and discussed.

\section{Image Binarization}

Figure 6 provides the variation in calculated basis weight as a function of threshold value. As can be seen, the optimum threshold value that provides a basis weight matching the physical value of $59 \mathrm{~g} / \mathrm{m}^{2}$ can easily be identified, in this case 103 . The binarized volume is a 3D dataset, $\boldsymbol{A}$, consisting of voxels labeled either 1 (fibre walls) or 0 (air and lumens).

\section{Lumen Tracking}

The hollow inside the fibres were tracked using the new methodology, resulting in dataset $\boldsymbol{B}$ consisting of 4348 lumens. Figure 7 (A) shows a single 2D cross-sectional image of the binarized fibres, along with the segmented lumens, i.e. $\boldsymbol{B}$ overlaid on $\boldsymbol{A}$. The gray regions correspond to the fibre walls from $\boldsymbol{A}$, while the coloured blobs represent segmented lumens from $\boldsymbol{B}$. It can be seen that the lumens with a wide variety of sizes and shapes and pores have been segmented.

The tracking of a single lumen through $2 \mathrm{D}$ slices in the presence of pores is shown in Figure 8 (A-D). A continuous fibre wall is shown in (A). Subsequently, a pore is initiated in the fibre wall as shown in (B), extending for approximately $4 \mu \mathrm{m}$ to $(\mathrm{C})$. The continuous fibre wall is 
regained in (D). As can be seen in (B) and (C), the lumen shape is not captured completely in the region of the pore and thus the segmented shape deviates from the actual shape. This is a direct consequence of the fact that the intersection of the background with the lumen shape in (D) is considered as the lumen in (B) and (C). However, by use of this method, the continuity of the lumen is not lost as the lumen continues to grow normally after the pore ends.

\section{Fibre Walls Extraction}

Based on dataset $\boldsymbol{B}$, the fibre walls surrounding the segmented lumen were then extracted, resulting in dataset $\boldsymbol{C}$ and comprising of 4348 segmented fibres. The fraction of fibre walls in $\boldsymbol{A}$ which have been segmented into individual fibres in $\boldsymbol{C}$ is $36 \%$. The low fraction is attributed to the collapsed fibres that cannot be segmented by the lumen tracking algorithm. Figure 7 (B) shows a single $2 \mathrm{D}$ cross-sectional image of the binarized fibres, along with the segmented fibre walls, i.e. $\boldsymbol{C}$ overlaid on $\boldsymbol{A}$. The corresponding 3D rendering of $\boldsymbol{C}$ is given in Figure 9 (a video further illustrating the final 3D rendering is provided in the on-line content). As can be seen, the proposed segmentation method preserves the true fibre wall thickness, and the points of intersection between fibres are clearly defined. The efficiency of the fibre wall labeling, however, depends on the accuracy of lumen tracking. Figure $8(\mathrm{E}-\mathrm{H})$ shows the result of fibre wall labeling on the when a pore is present. It can be seen that the fibre wall is identified even at the location of the pore by the multi-step fibre wall extraction method. However, as shown in Figure $8(\mathrm{G})$, the presence of the pore can result in poor fibre wall segmentation. By comparing (G) and (C), it is clear that when the lumen shape is not completely captured, the resulting fibre wall is also only partially selected.

\section{Second Level Segmentation}

The lumen tracking and fibre walls labeling steps resulted in 4348 fibres being uniquely identified within the 3D tomographic image, a large number of the lumen appear to be quite small, as previously shown in Figure 4. This is because the methodology for lumen tracking is 
unable to trace lumens through collapsed sections of fibres and hence splits the lumen into small fragments. The information contained in datasets $\boldsymbol{A}, \boldsymbol{B}$ and $\boldsymbol{C}$ was then utilized by the second level segmentation process to both label the collapsed sections and merge fibre fragments, resulting in dataset $\boldsymbol{D}$. By merging the fibres and including segmentation of collapsed sections, the total number of fibres was reduced to 2484 , and the fraction of total fibre voxels that were segmented increased to 40\%. As shown in Figure 4, the second level segmentation is able to capture the whole fibre as a single segmented object. Due to the merging of fibres via the second level segmentation, the fraction of fibres shorter than 100 microns decreased from $86 \%$ in dataset $\boldsymbol{C}$ to $64 \%$ in $\boldsymbol{D}$ while the fraction of fibres shorter than 200 microns decreased from $95 \%$ to $84 \%$. In total, dataset $\boldsymbol{D}$ contained 87 fibres that were longer than 500 microns, while dataset $\boldsymbol{C}$ contained only 20 fibres of similar length.

As shown through the above analysis, the new fibre segmentation methodology has segmented tortuous fibrous material within a 3D dataset in a robust and efficient manner while conserving fibre topology. The main drawback to the algorithm, for papermaking fibres, is that only $40 \%$ of the fibre walls have been labeled. This is due to the fact that the completely collapsed fibres, and fibre fragments, cannot be segmented using this methodology. Nevertheless, the methodology significantly advances our ability to segment complex fibres on an individual basis.

\subsection{Characterization of Fibre Properties}

The lengths and coarseness of the segmented fibres was calculated using the methods presented in section 2.3.3. Figure 10 shows the distributions of the fibre lengths (fibre fragments smaller than 100 microns have not been considered) after the second level segmentation process. It can be seen that fibres have been traced through the $3 \mathrm{D}$ image for as long as $1.8 \mathrm{~mm}$. The coarseness value was also calculated only for fibres longer than 100 microns. Figure 11 shows the probability distribution of the coarseness value of 895 fibres found in dataset $\boldsymbol{D}$. The mode 
value of coarseness values is found to be $0.13 \mathrm{mg} / \mathrm{m}$ while the mean is $0.16 \pm .06 \mathrm{mg} / \mathrm{m}$ as compared to the physically measured value of approximately $0.12 \mathrm{mg} / \mathrm{m}$ for the NBSK pulp.

Although the second level segmentation resulted in a significant improvement in the result, a large number of small fibres remain individually segmented. These represent a large number of tiny fragments created due to noise in the dataset. Moreover, the average fibre length for the non-fractionated NBSK pulp used to manufacture the handsheet is $2.5 \mathrm{~mm}$ and hence the

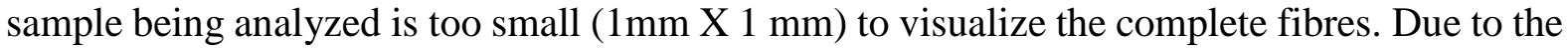
small sample size, the segmented fibres provide only a partial representation of NBSK fibres, resulting in a poor estimate of the coarseness.

\section{Conclusion}

The fibre segmentation algorithm presented in this paper is the first method that is able to segment a large fraction (40\%) of papermaking fibres within the dense network structure of paper handsheets in an automated manner. The algorithm is able to robustly capture the true surface topology as well as the tortuous morphology of papermaking fibres despite the numerous complexities present in the data. Moreover, it is considerably faster than the Ordered Region Growing method of Aronsson (2002), which was able to segment only 10-20\% fibres in a semi-automatic manner.

Applying shape constraints on the connected components that are used to initiate lumens can significantly reduce the number of false positives. Methods for selecting potential lumen candidates on the basis of shape constraints presented by Lunden (2002) could be leveraged in future work. Furthermore, methods can be developed to directly segment the walls of collapsed fibres in a simplified dataset created by removing the segmented voxels from $\boldsymbol{A}$. The segmented dataset can be used to measure fibre level properties such as fibre curl, torsional resistance and slenderness ratio. Further, the conversion of the segmented network to a graph will enable the measurement of network level properties such as free fibre length and coordination number of 
fibre-fibre contacts. Also, fibre properties can be measured accurately by using a larger sample size obtained by combining scans of adjacent sections.

\section{Acknowledgement}

The authors would like to thank Canfor Pulp Products Inc., the Natural Sciences and Engineering Research Council of Canada (NSERC) and MITACS for funding this work. 


\section{References}

Aronsson, M. (2002) On 3D fibre measurements of digitized paper. PhD thesis, Centre for Image Analysis, Swedish University of Agricultural Sciences, Uppsala, Sweden.

Adams, R. \& Bischof, L. (1994) Seeded region growing. IEEE Transactions on Pattern Analysis and Machine Intelligence (PAMI), 16 (6), 641-647.

Axelsson M. (2007) 3D tracking of cellulose fibres in volume images. 14th International Conference on Image Processing, pp. IV 309-312, San Antonio, Texas, USA.

Bache-Wiig, J. \& Henden, P.C. (2005) Individual fiber segmentation of three dimensional microtomograms of paper and fiber-reinforced composite materials. Master's thesis, Department of Computer and Information Science, Norwegian University of Science and Technology, Trondhiem, Norway.

Donoser, M. \& Bischof, H. (2006) Efficient maximally stable extremal region (MSER) tracking. IEEE Computer Society Conference on Computer Vision and Pattern Recognition, vol. 1, pp. 553-560. doi: 10.1109/CVPR.2006.107

Donoser, M., Riemenschneider, H., \& Bischof, H. (2009) Efficient partial shape matching of outer contours. Lecture Notes in Computer Science. 5994, 281-292. 
Kontscheider P., Donoser, M., Bischof, H., Kritzinger, K. \& Bauer,W. (2010) Detecting paper fibre cross sections in microtomy images. $20^{\text {th }}$ International Conference on Pattern Recognition (ICPR), pp. 316-319. doi: 10.1109/ICPR.2010.86

Lu, C., Latecki, L.J., Adluru, N., Yang, X. \& Haibin L. (2009) Shape guided contour grouping with particle filters. IEEE $12^{\text {th }}$ International Conference on Computer Vision (ICCV), pp. 22882295, Sept. 29-Oct. 2 2009, doi:10.1109/ICCV.2009.5459446.

Lunden, J. (2002) Image analysis methods for evaluation of fibre dimensions in paper crosssections. Master's thesis, The Swedish Royal Institute of Technology, Uppsala, Sweden.

Lux, J., Delisee, C. \& Thibault, X. (2006) 3D characterization of wood based fibrous materials: an application. Image anal stereol, 25 (1), 25-35.

Matas, J., Chum, O., Urban, M. \& Pajdla, T. (2004) Robust wide baseline stereo from maximally stable extremal regions. Image and Vision Computing, 22 (10), 761-767.

Sandau, K., \& Ohser, J. (2007) The chord length transform and the segmentation of crossing fibres. Journal of Microscopy, 226 (1), 43-53.

Seber, G.A.F. (1984) Multivariate Observations, John Wiley \& Sons, Inc., Hoboken, NJ.

Sezgin, M. \& Sankur, B. (2004) Survey over image thresholding techniques and quantitative performance evaluation. Journal of Electronic Imaging, 13 (1), 146-165. 
Svensson, S. \& Aronsson M. (2003) Using distance transform based algorithms for extracting measures of the fiber network in volume images of paper. IEEE Transactions on Systems, Man, and Cybernetics-Part B, 33 (4), 562-571.

Udupa, J.K. \& Samarasekera S. (1996) Fuzzy connectedness and object definition: Theory, algorithms, and applications in image segmentation. Graph. Models Image Process., 58, 246261.

Weickert, J., Romeny, B.M.T.H. \& Viergever, M.A., (1998) Efficient and reliable schemes for nonlinear diffusion filtering. IEEE Transactions on Image Processing, 7 (3), 398-410, doi: $10.1109 / 83.661190$

Wernersson, E. L. G., Brun, A. \& Hendriks, C. (2009) Segmentation of wood fibres in 3D CT images using graph cuts. 15th International Conference on Image Analysis and Processing, pp: 92-102, Springer-Verlag.

Yang, H. \& Lindquist W. (2000) Three-dimensional image analysis of fibrous materials. Applications of Digital Image Processing, pp. 275-282, San Diego, Calif. 


\section{Figure Captions}

Figure 1: Microstructure of paper acquired using X-ray $\mu \mathrm{CT}$ A) $3 \mathrm{D}$ volume B) $2 \mathrm{D}$ image sliced along the $\mathrm{x}-\mathrm{y}$ plane as shown by the clipping plane; the sample rotation is also indicated.

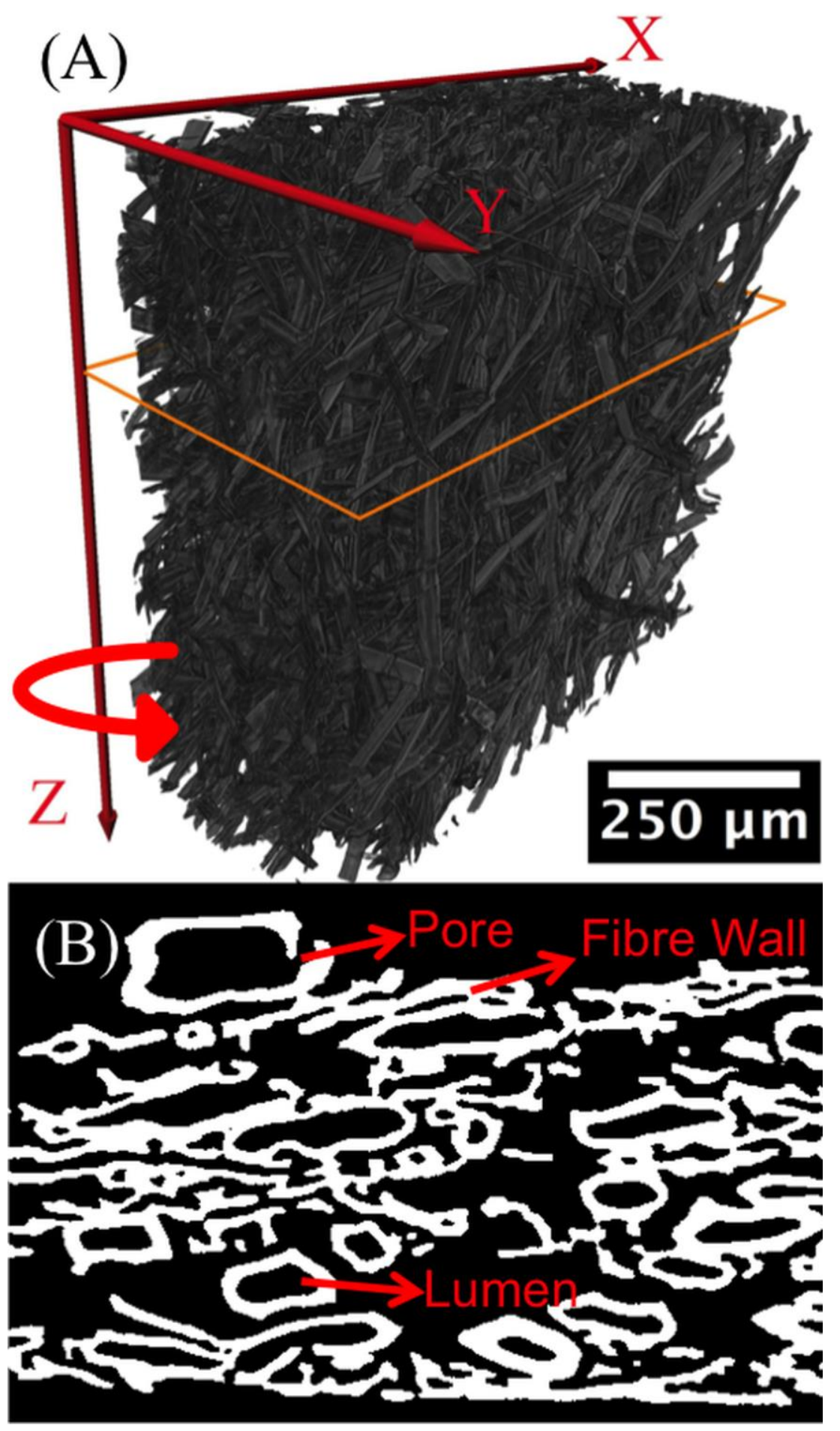


Figure 2: Lumen cross-sections in successive slices showing the tracking algorithm; the colored blobs are the result of connected component labeling and are merged in successive slices to build the 3D lumen

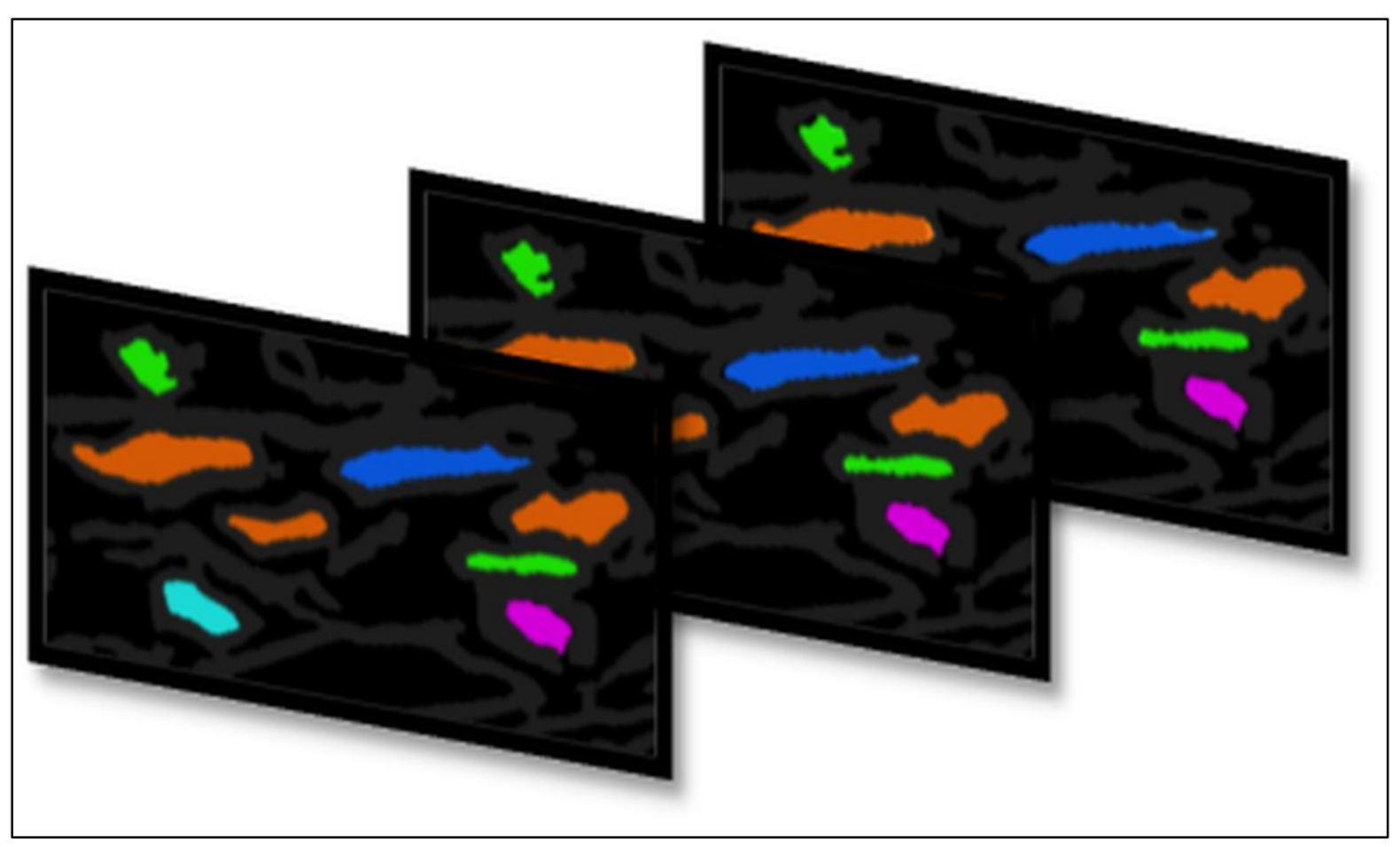


Figure 3: A) Connected component labeling on a 2D slice of tomography data; the lumens with pores in their walls get merged with the background B) The same section after the lumen segmentation; the lumen previously merged with the background has been segmented out
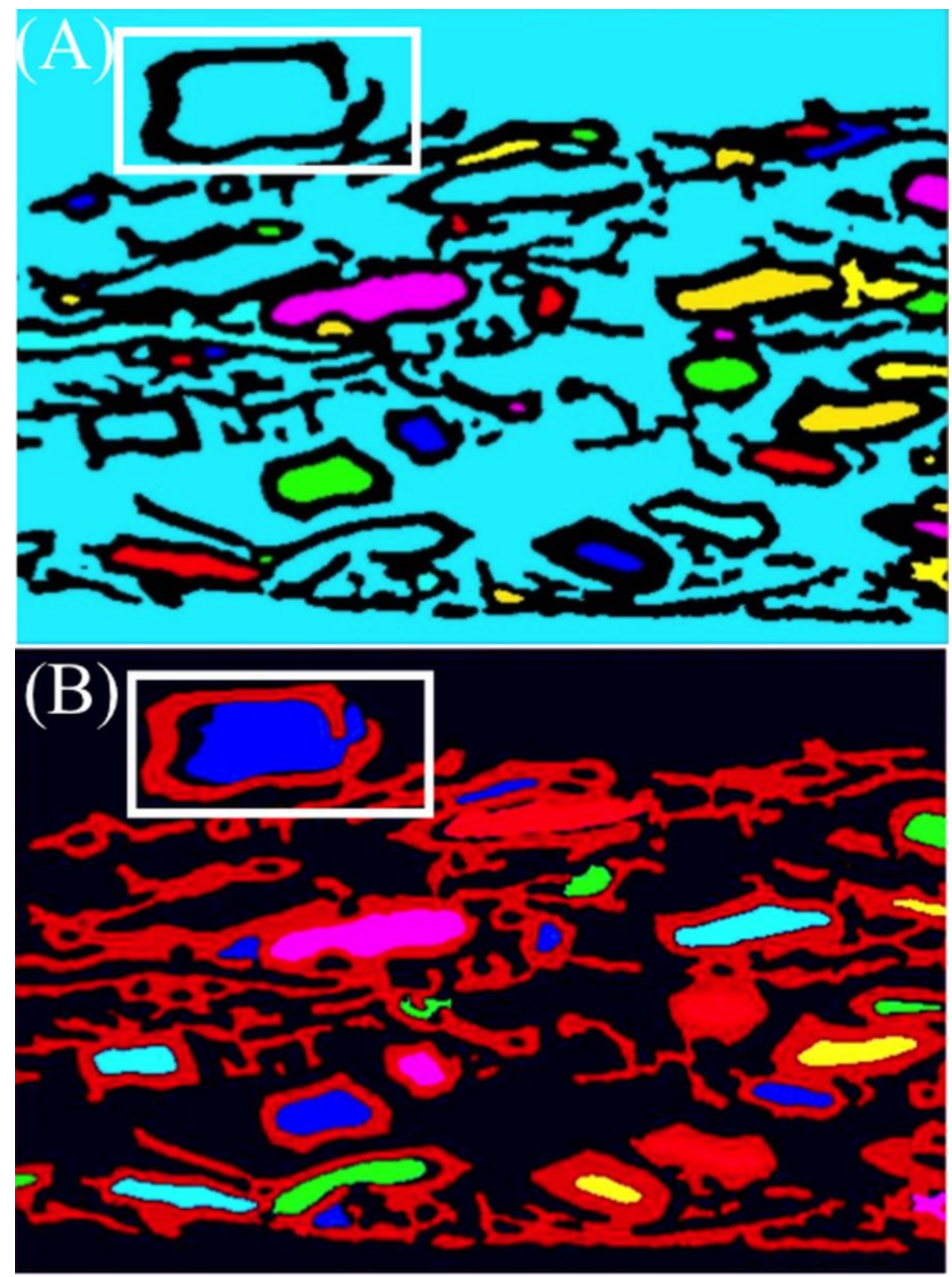
Figure 4: Example of a fibre split into 4 fragments and later merged by the second level segmentation A) Lumen split in 4 section (A,B,C and D) in dataset $\boldsymbol{B}$ B) Corresponding fibre walls in dataset $\boldsymbol{C}$ along with the un-segmented i.e. the collapsed regions C) Merged fibre in dataset $\boldsymbol{D}$

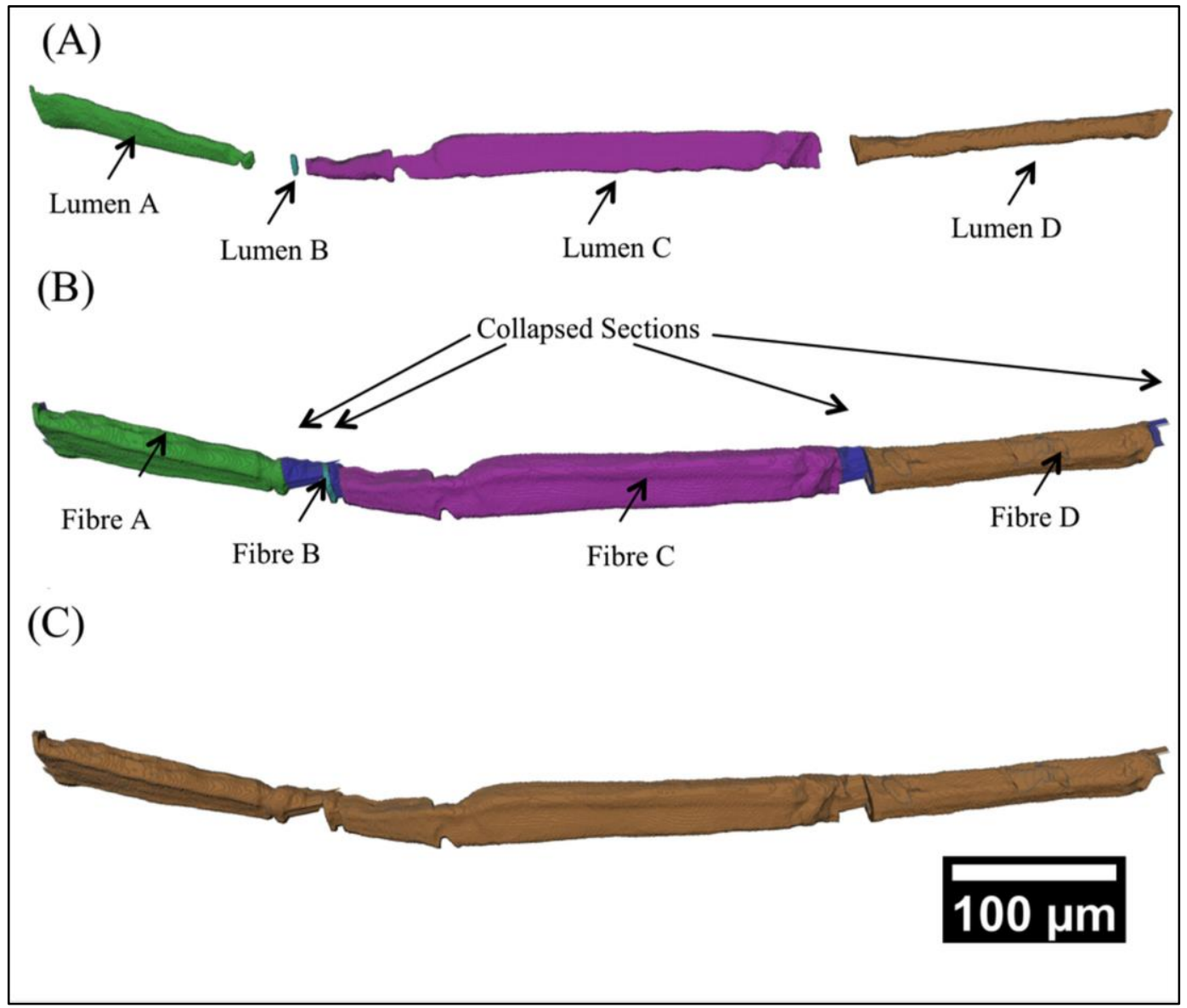


Figure 5: A) Test geometry B) Papermaking fibres simulated by bent and porous aluminum tubes C) 2D slice of acquired data; there is good contrast in the images; the discontinuities in the fibre walls and incomplete cross-sections of out of plane tubes can be seen D) Result of fibre segmentation
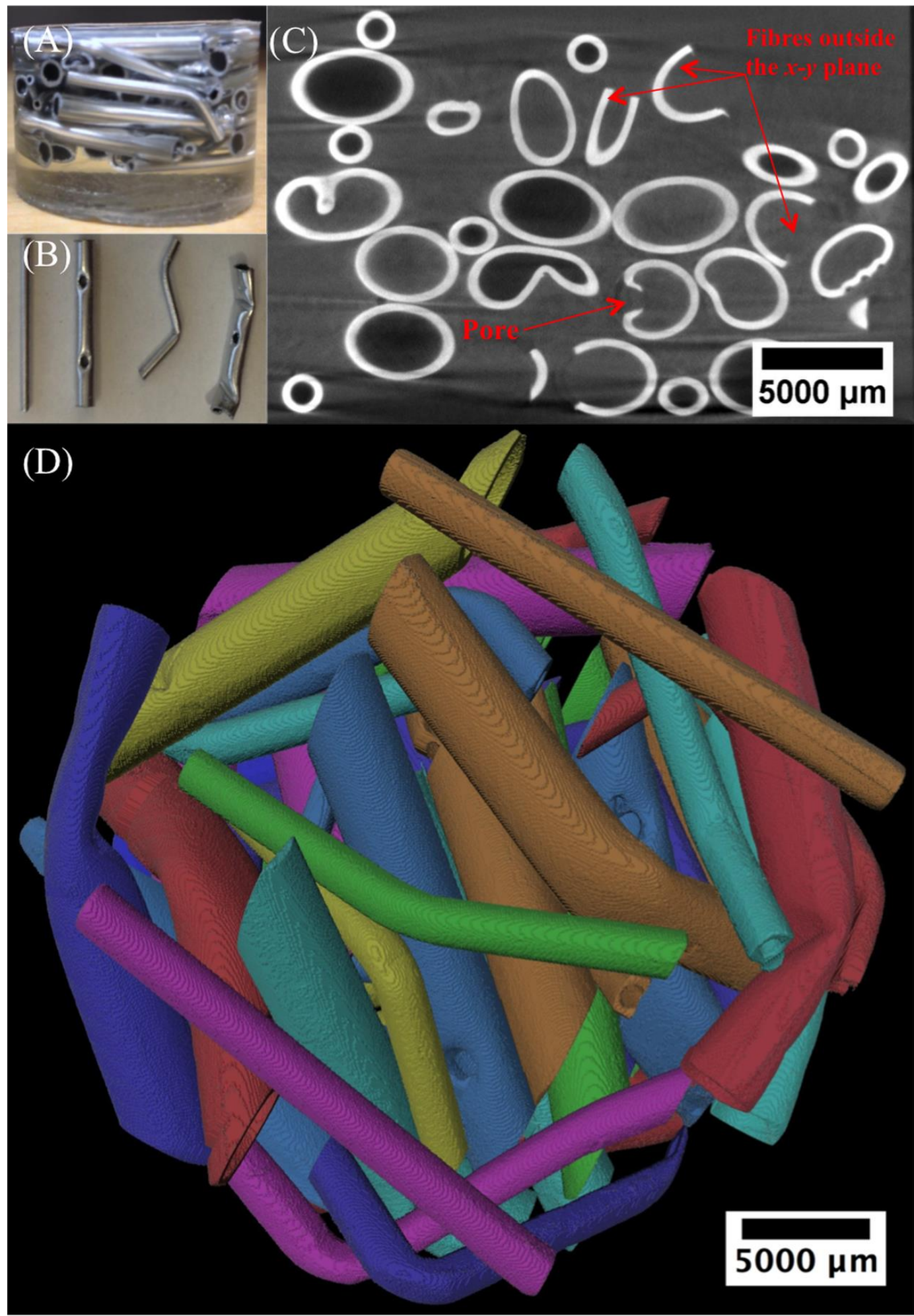
Figure 6: Calculated value of basis weight as a function of the binarization threshold

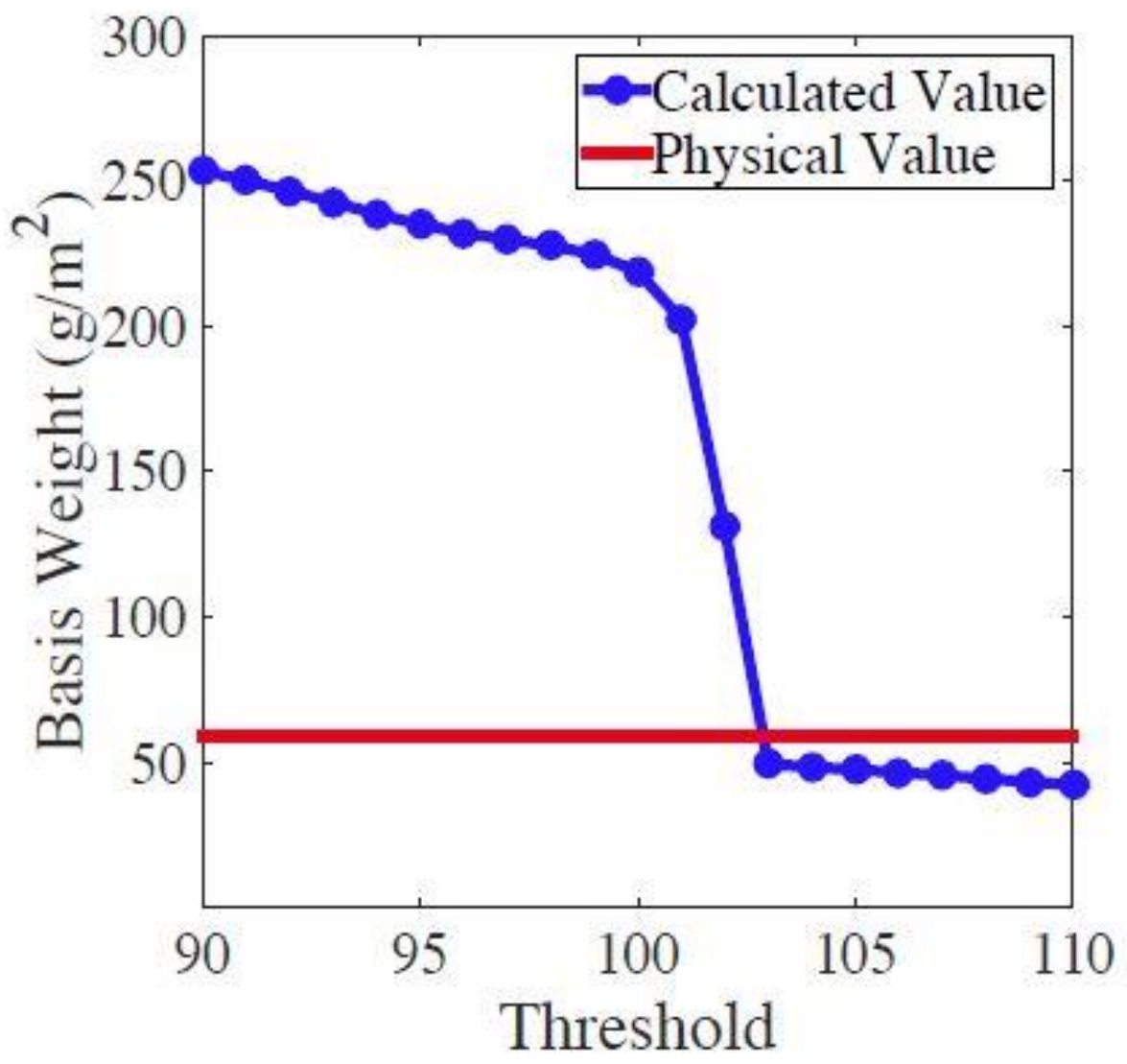


Figure 7: A) 2D slice showing the segmented lumens $(\boldsymbol{B})$ overlaid on the binary data $(\boldsymbol{A}) \mathrm{B})$ 2D slice showing the segmented fibre walls $(\boldsymbol{C})$ overlaid on the binary data $(\boldsymbol{A})$

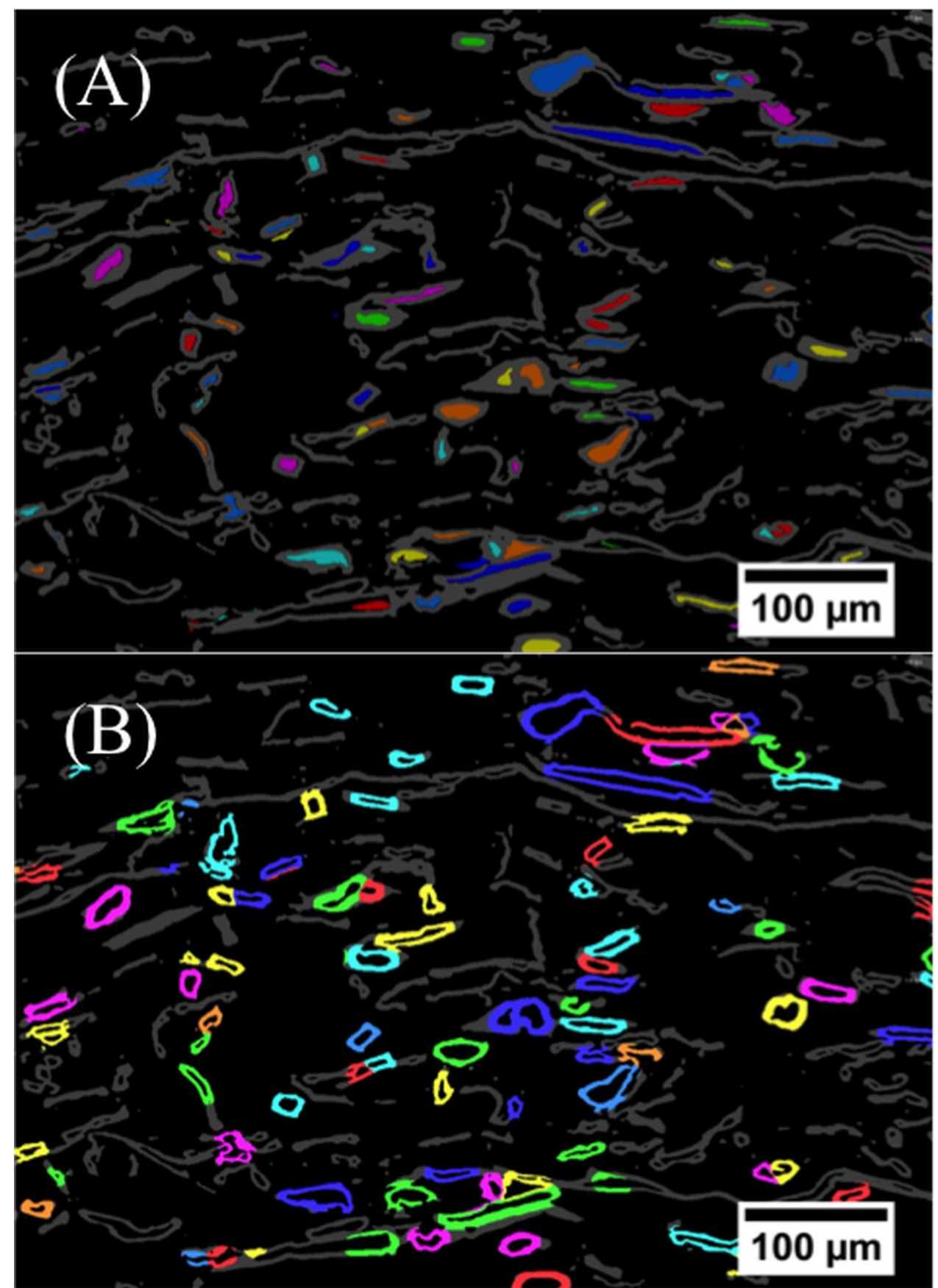


Figure 8: The process of tracking lumen in presence of a crack is shown. The result of lumen segmentation is shown in images (A-D) on the left side with the corresponding result of fibre wall segmentation on the right side $(\mathrm{E}-\mathrm{H})$. The gray regions represent the original fibre walls while the colored regions represent the segmented lumens

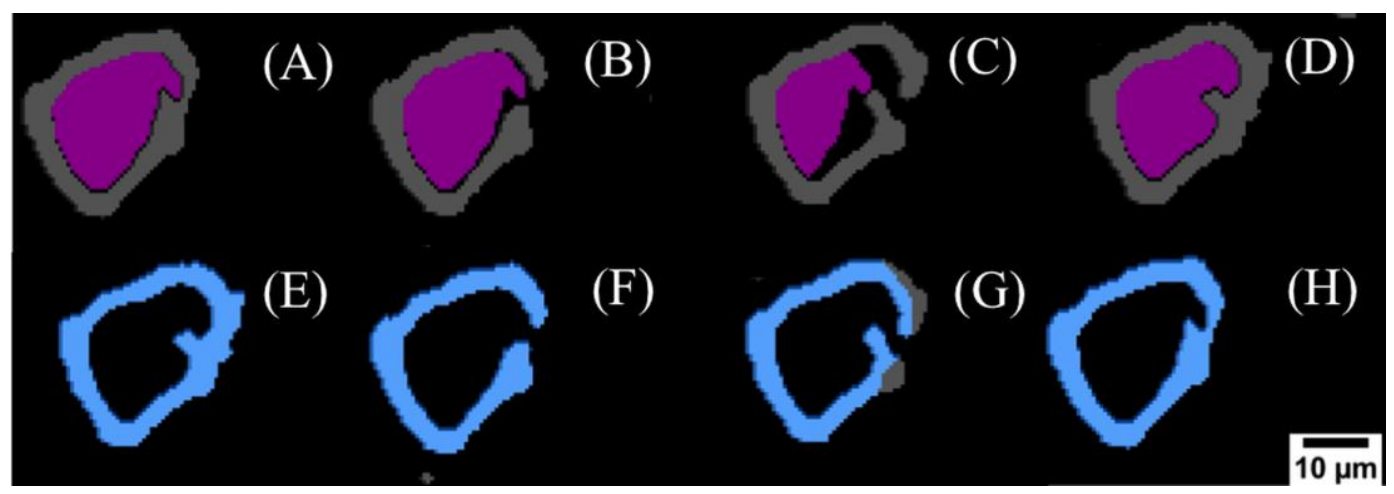

Figure 9: 3D rendering of dataset $\boldsymbol{C}$, each segmented fibre is assigned a unique label.

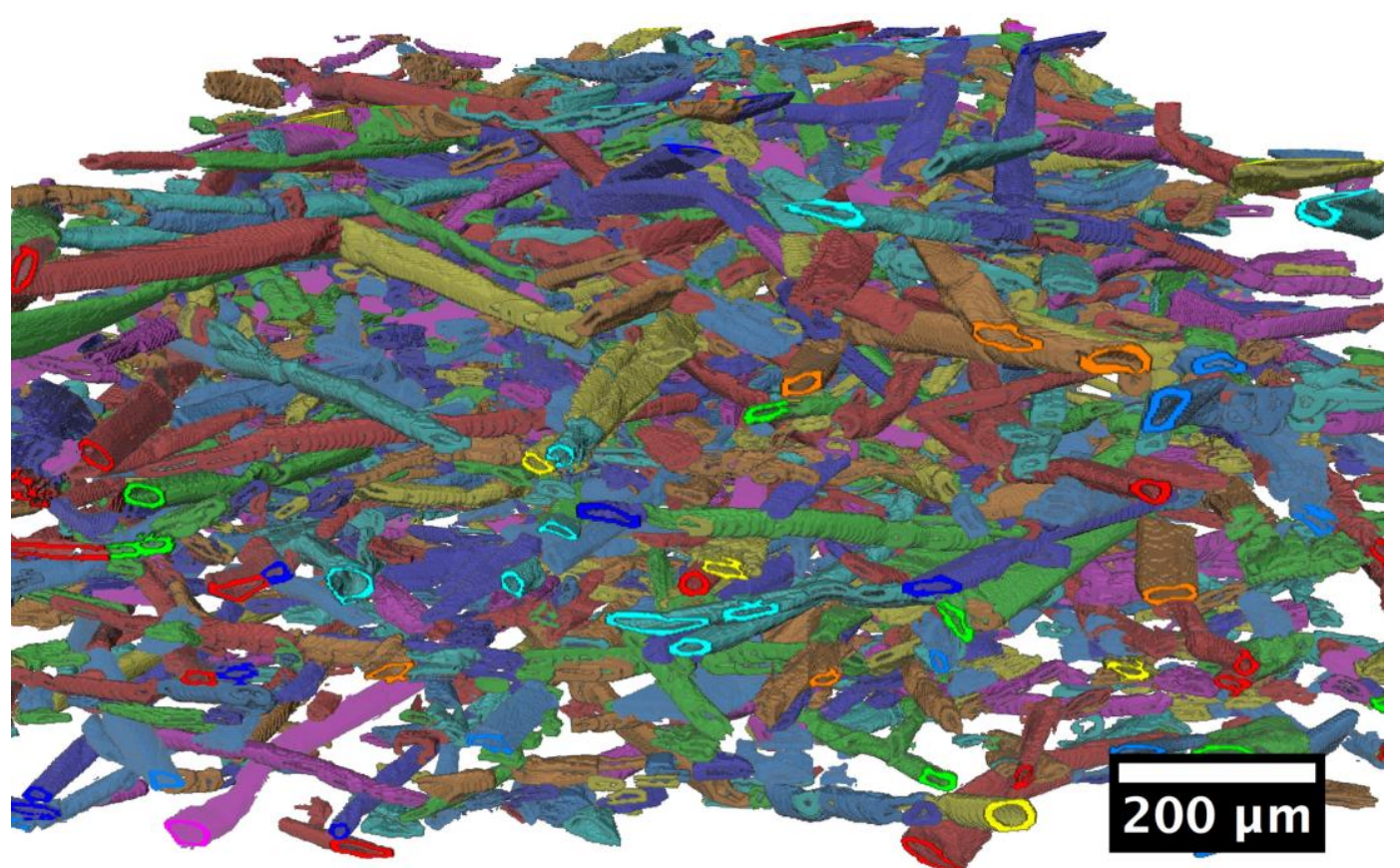


Figure 10: Distribution of fibre lengths in dataset $\boldsymbol{D}$.

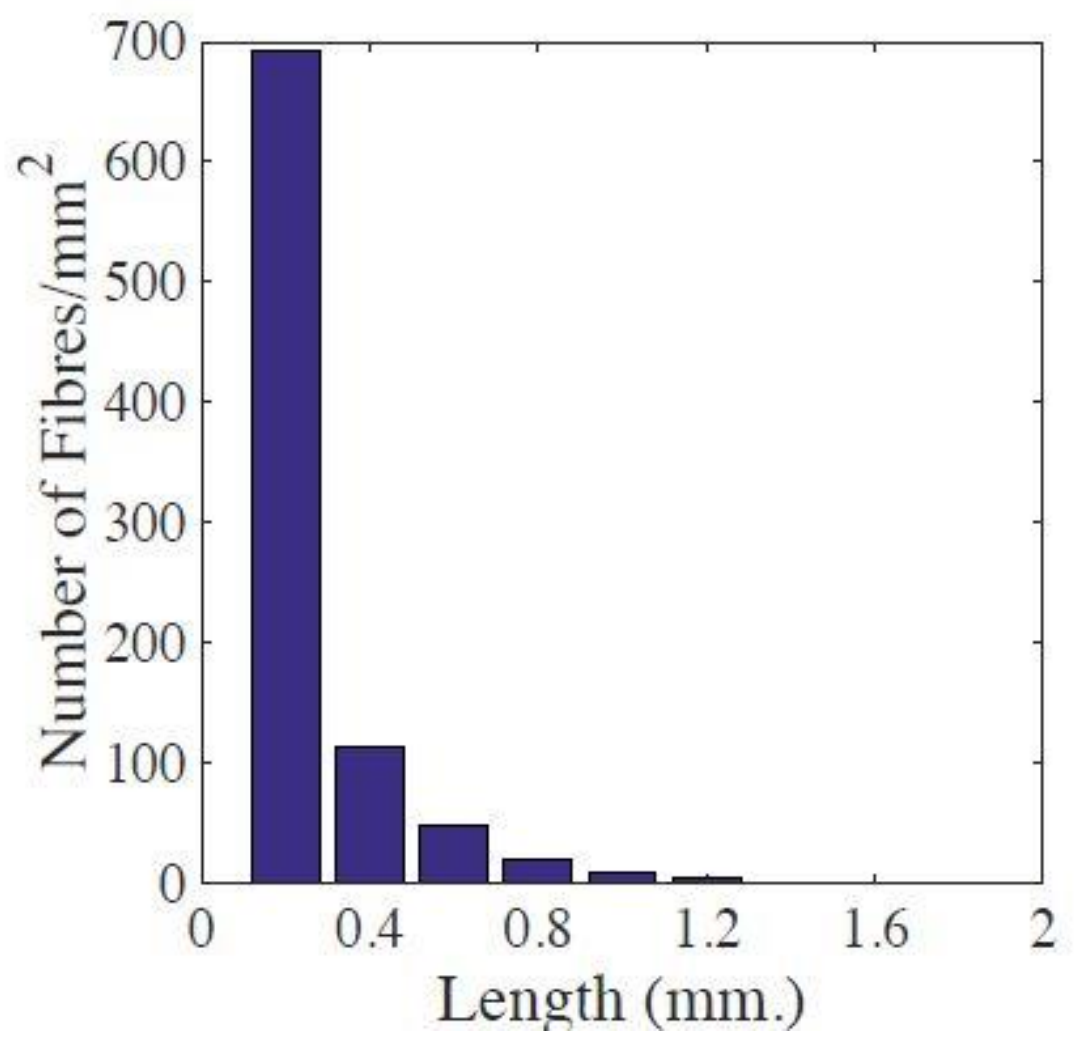

Figure 11: Probability distribution of fibre coarseness in dataset $\boldsymbol{D}$

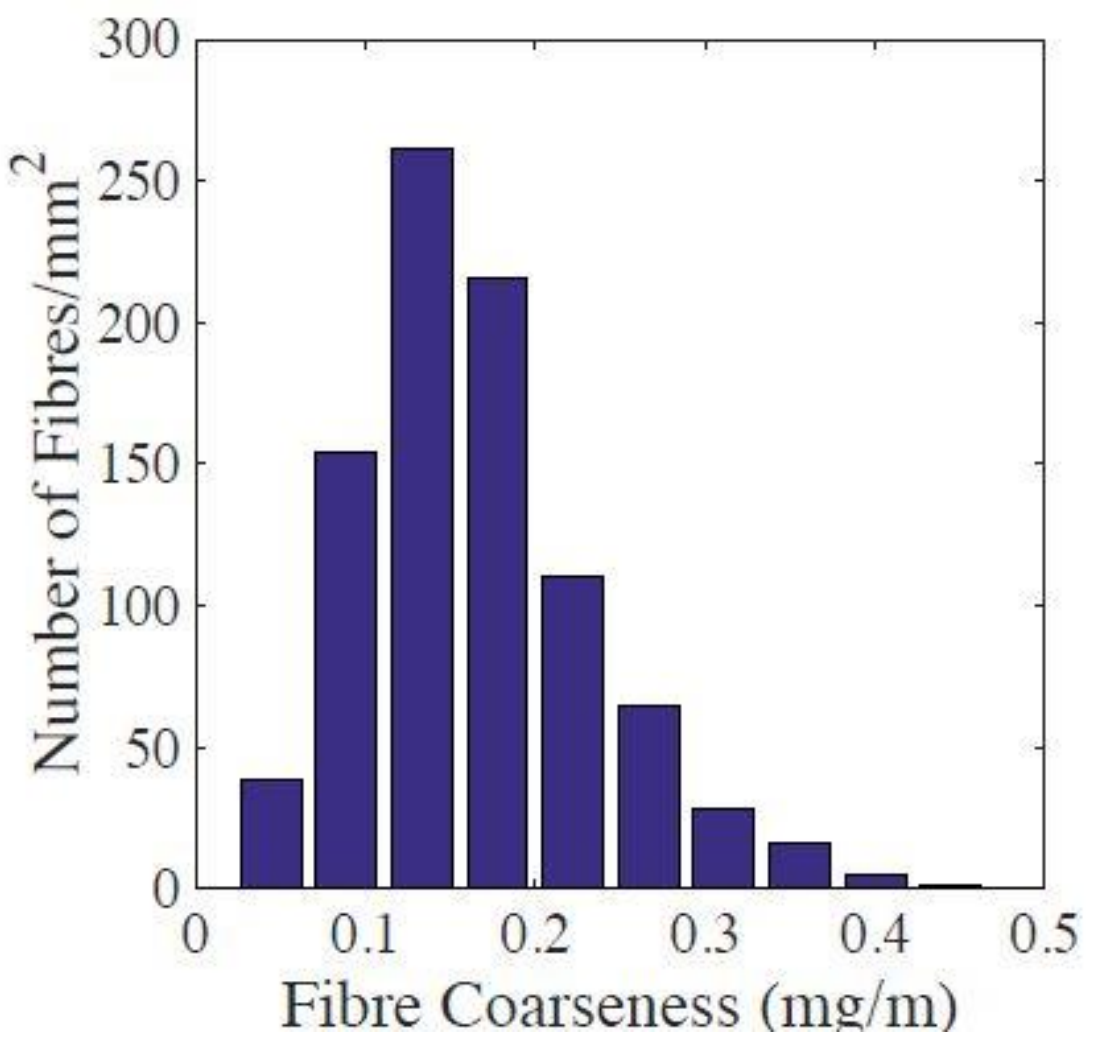

